\title{
Mortality, functional outcomes and quality of life after hip fractures complicated by infection: a case control study
}

\author{
Antonios A. Koutalos, Christos Baltas, Vasileios Akrivos, Christina Arnaoutoglou, and \\ Konstantinos N. Malizos \\ Department of Orthopaedic Surgery \& Musculoskeletal Trauma, Faculty of Medicine, \\ University of Thessaly, Larissa, Greece \\ Correspondence: Antonios A. Koutalos (akoutmed@gmail.com)
}

Received: 19 June 2021 - Revised: 3 September 2021 - Accepted: 6 September 2021 - Published: 23 September 2021

\begin{abstract}
Introduction: Infection is a detrimental complication of operatively treated hip fractures. The objective of this retrospective case-control study was to evaluate the mortality, the physical function and the quality of life of hip fractures complicated with infection and determine risk factors for deep infection in hip fractures. Patients and methods: All patients with hip fractures (31A and 31B OTA/AO) that were operatively managed over a 10-year period that subsequently developed deep infection were included in the study. Thirty-nine patients met the inclusion criteria. These patients were compared with a matched control group of 198 patients without infection. Minimum follow-up was 1 year. Mortality, Barthel index score, EQ-5D-5L, Parker mobility score and visual analogue scale (VAS) pain score were compared between groups. Results: Mortality at 1 month was $20.5 \%$ and $43 \%$ at 1 year. Half of the infections were acute and $28 \%$ were polymicrobial. Mortality was greater in the infection group ( $43 \%$ vs. $16.5 \%, p<0.0014$ ), and Barthel index was inferior in the infection group (14 vs. $18, p<0.0017)$ compared to control group. Logistic regression analysis revealed that time from admission to surgery was a negative factor that predisposed to infection. Conclusions: Patients complicated with infection after a hip fracture have higher mortality and inferior functional results. Delay from admission to surgery predisposes to infection.
\end{abstract}

\section{Introduction}

Fragility hip fractures are associated with significant limitation in physical activity and mortality among the elderly patients (Johnell and Kanis, 2004). In addition to this, infection is a dreadful complication with negative effects on function and mortality (Partanen et al., 2006). The incidence of fracture-related infection (FRI) after hip fixation surgery is reported between $1.2 \%$ and $3.6 \%$ (Pollard et al., 2006; Edwards et al., 2008). Diabetes mellitus, steroids treatment and operative time have been shown by several studies to increase the risk of infection (Partanen et al., 2006; Edwards et al., 2008).

The FRIs after hip fractures are devastating for the patient and their family, require multiple operations, antibiotics, increased length of stay $(\operatorname{LoS})$ in the hospital, office visits and much longer rehabilitation (Pollard et al., 2006; Edwards et al., 2008). The current protocols involve patient optimisation of the general health and nutritional status, surgical management without delay, control of blood loss and early mobilisation after hip fracture fixation, to diminish the risk of complications and infection.

The purpose of the study was (1) to explore any risk factors that predispose to deep infection after hip fracture surgery, (2) to examine the impact of infection on mortality, and (3) to evaluate the final functional outcomes. The hypothesis is that patients with infections would have higher mortality and lower functional status.

\section{Patients and methods}

This was a case control study of geriatric patients with hip fractures that underwent surgical management and were complicated by infection. From 2010 to 2020, 5453 hip fractures were surgically managed in a teaching centre. In the 
same period 39 patients were complicated with FRI and were treated accordingly. Inclusion criteria included cases with deep infection, diagnosed after operative fixation of a hip fracture (31A and 31B according to OTA/AO classification) and a minimum follow-up of 1 year.

Exclusion criteria were hip surgery performed for reasons other than fracture, patients treated for the hip fracture in other hospitals and were referred to our tertiary centre for infection management, hip fractures that were managed conservatively, and superficial infections or skin ulcers. Patients younger than 65 were also excluded because the main focus of our study was elderly patients having low-energy hip fractures. These patients were compared with a subset of patients that had hip fracture and have been part of the prospectively maintained local database since 2018. Epidemiological, clinical, radiographic, and pre- and postoperative functional scores are stored in the intra-departmental database.

Diagnosis of infection was established if two or more deep intraoperative cultures were positive, in the presence of a sinus communicating with the hip, in the occurrence of wound breakdown or the presence of pus coming beneath the fascia (Metsemakers et al., 2018).

A management plan was agreed on by the multidisciplinary team composed of an orthropaedic surgeon, an infection disease specialist and a geriatrician/internal medicine specialist. Host optimisation involved correction of malnutrition and hyperglycemia if present. Serial debridement, antibiotics and implant retention (DAIR), antibiotic-loaded bone cement spacers (ALBCS) and vacuum-assisted closure (VAC) devices were implemented to eliminate infection, together with IV antibiotics for 6 weeks. An attempt was made for implant retention in the acute infections (diagnosed < 6 weeks) until fracture healing occurred. On the contrary, implants were removed in late infections (diagnosed $\geq 6$ weeks) with healed fracture, in unstable implants or fractures, and in immunocompromised patients (host $\mathrm{C}$ according to Cierny) (Cierny et al., 2003). Follow-up after discharge included outpatient clinic visits at 3, 6 and 12 weeks for clinical examination and radiographs if needed and then annually outpatient visits.

Demographics were recorded (Table 1). Patient information, including smoking, accommodation place involving geriatric centres or own residence, the presence of dementia, diabetes mellitus, if they walked with or without assistance, the use of anti-coagulants, haemoglobin and albumin levels at admission, was retrieved from the medical notes. Pre-operative function was assessed with the Barthel index.

Data regarding fracture type (extra- or intra-capsular), type of surgery performed, length of stay in the first admission, total length of stay combining all admissions, ASA score (Owens et al., 1978), delay till the operation after admission, mean operative time, number of debridements and if implants were finally removed or not were collected from the medical records. Complications during hospital stay were also recorded. Information on 30-day and 1-year mortality, the length of follow-up, the time of infection diagnosis from operation, the bacteria responsible for the infection, Gram stain, and mono- or polymicrobial infection was also documented.

In the last follow-up, patients were interviewed at the outpatient clinic or over telephone questionnaire by a researcher not involved in their management. Visual analogue scale (VAS) pain score, Parker mobility score (Parker and Palmer, 1993), Barthel index (Mahoney and Barthel, 1965) and EQ5D-5L (Herdman et al., 2011) utility index were assessed. Parker mobility measures the mobility of the patient indoors, outdoors and during shopping and is often used as an outcome measure in hip fractures or as a predictor of mortality (Kristensen et al., 2010). Barthel index evaluates the ability to perform basic activities of daily living and has been widely utilised in geriatric patients. It has been validated in hip fractures (Bouwstra et al., 2019; Mayoral et al., 2019). Minimal clinical important difference is calculated to three points $(\mathrm{Xu}$ et al., 2019). EQ-5D-5L measures the quality of life with a utility index ranging from 0 (death) to 1 (perfect health). The average utility after hip fracture varies from 0.379 at 4 months to 0.67 at 24 months (Polinder et al., 2007; Sims et al., 2018). The minimum clinical important difference is 0.08 (Walters and Brazier, 2005).

\subsection{Control group}

The control group consisted of 210 consecutive patients with hip fractures operated from January to June 2018. Twelve patients were lost to follow-up. Minimum follow-up was 1 year. The same demographics, clinical data and information regarding the fracture, the surgery and complications are recorded in these patients. Pre- and postoperative Barthel index score was documented. Postoperative functional level evaluation including Parker mobility score and quality of life data involving EQ-5D-5L index are also available for these patients. Mortality at 1 month and at 1 year was also calculated.

\subsection{Outcomes}

The primary outcome was the mortality rate and the functional outcome as measured with the Barthel index score. Secondary analysis included the examination of whether infection influences the final Barthel index score and the search for predisposing factors of infection after hip fractures.

\subsection{Statistics}

Descriptive statistics were used for demographics. Normality of the data was evaluated with the Shapiro-Wilk test. For normally distributed data, mean $\pm \mathrm{SD}$ was presented, while for skewed data, median \pm interquartile range (IQR) was used. $T$ test was utilised for comparing groups of normally distributed data and the non-parametric Mann-Whitney $U$ test otherwise. Chi-squared test was used for categorical data 
Table 1. Demographics of the group of patients that complicated with infection after fracture fixation.

\begin{tabular}{|c|c|}
\hline \multicolumn{2}{|l|}{ Sex } \\
\hline Male $[n(\%)]$ & $8(21 \%)$ \\
\hline Female $[n(\%)]$ & $31(79 \%)$ \\
\hline Age [mean (SD)] & $79(13)$ \\
\hline \multicolumn{2}{|l|}{ Smoking } \\
\hline Yes $[n(\%)]$ & $8(21 \%)$ \\
\hline No $[n(\%)]$ & $31(79 \%)$ \\
\hline \multicolumn{2}{|l|}{ Accommodation } \\
\hline Home $[n(\%)]$ & $37(95 \%)$ \\
\hline Not living in own home $[n(\%)]$ & $2(5 \%)$ \\
\hline \multicolumn{2}{|l|}{ Dementia } \\
\hline Yes $[n(\%)]$ & $5(13 \%)$ \\
\hline No $[n(\%)]$ & $34(87 \%)$ \\
\hline \multicolumn{2}{|l|}{ Diabetes mellitus } \\
\hline Yes $[n(\%)]$ & $11(28 \%)$ \\
\hline No $[n(\%)]$ & $28(72 \%)$ \\
\hline \multicolumn{2}{|l|}{ Mobilisation without assistance } \\
\hline Yes $[n(\%)]$ & $33(85 \%)$ \\
\hline No $[n(\%)]$ & $6(15 \%)$ \\
\hline \multicolumn{2}{|l|}{ Use of anti-coagulants } \\
\hline Yes $[n(\%)]$ & $21(54 \%)$ \\
\hline No $[n(\%)]$ & $18(46 \%)$ \\
\hline Haemoglobin at admission [mean (SD)] & $11.5(2.4)$ \\
\hline Albumin at admission [mean (SD)] & $3.6(0.8)$ \\
\hline ASA score [mean (SD)] & $2.5(0.2)$ \\
\hline \multicolumn{2}{|l|}{ Fracture type } \\
\hline Extra-capsular $[n(\%)]$ & $25(64 \%)$ \\
\hline Intra-capsular $[n(\%)]$ & $14(36 \%)$ \\
\hline Length of stay on first admission (days) (LOS) [median (IQR)] & $12(31)$ \\
\hline Length of stay combining all admissions (days) (LOS) (mean, SD) & $37(8)$ \\
\hline Operative time (hours) [median (IQR)] & $1.4(0.6)$ \\
\hline Number of operating room visits for debridement [median (IQR)] & $2(2)$ \\
\hline \multicolumn{2}{|l|}{ Fate of implants } \\
\hline Removed $[n(\%)]$ & $16(41 \%)$ \\
\hline Not removed $[n(\%)]$ & $23(59 \%)$ \\
\hline
\end{tabular}

analysis. Regression analysis was used for testing the hypothesis of whether infection had an effect on Barthel index score. Except infection, the variables tested included age, sex, smoking, dementia, diabetes mellitus, previous accommodation, previous mobilisation status, use of anticoagulants, haemoglobin, albumin, ASA score, fracture type, method of fixation, length of stay, operative time and waiting time until surgery. Finally, logistic regression analysis was employed for the search of any predisposing factors to infection among the same variables. SPSS statistics package v.25 (IBM, Armonk, New York, USA) was used for statistical analyses. 
Table 2. Micro-organisms isolated from infected hip fracture cases.

\begin{tabular}{lr}
\hline Micro-organism isolated & Frequency $[n(\%)]$ \\
\hline Gram positive & $6(15 \%)$ \\
\hline Staphylococcus aureus MSSA & $7(18 \%)$ \\
Staphylococcus aureus MRSA & $6(15 \%)$ \\
Staphylococcus epidermidis & \\
\hline Gram negative & $3(8 \%)$ \\
\hline Escherichia coli & $5(13 \%)$ \\
Acinetobacter baumannii & $11(28 \%)$ \\
\hline Polymicrobial infections & $4(10 \%)$ \\
Enterococcus faecalis + Acinetobacter & $3(7.5 \%)$ \\
baumannii & \\
Staphylococcus aureus MRSA + Pseu- & $3(7.5 \%)$ \\
domonas aeruginosa & $1(3 \%)$ \\
Staphylococcus aureus MRSA + Kleb- & \\
siella pneumoniae & \\
Staphylococcus aureus MRSA + Can- & \\
dida spp. & \\
Negative cultures &
\end{tabular}

\section{Results}

From 2010 to 2020, 5453 hip fractures received surgical treatment, of which 3435 were extra-capsular and 2018 were intra-capsular fractures (31A and 31B OTA/AO classification respectively). The infection rate over the entire period of observation was $0.72 \%$ (39 of 5453 hip fractures). Extracapsular fractures were complicated by infection in $0.69 \%$ of the cases (14 of 2018) and intra-capsular fractures in $0.72 \%$ (25 of 3435). The rate of infection after hemiarthroplasty for the intra-capsular fractures was $0.7 \%$, in the patients fixed with intramedullary nails $0.8 \%$ and $0.6 \%$ in patients with sliding hip screws. No infections were diagnosed after total hip arthroplasty or cannulated screws. Twenty infections were diagnosed from 2010 to 2014 and 19 from 2015 to 2020. Approximately half of the infections (20 of 39) were diagnosed in less than 6 weeks. The patients underwent a mean of two surgical debridement for eradication of the infection (range 1-5). With regard to the microbiology of the infection, gram-positive micro-organisms were isolated in $48 \%$ of cases with methicillin-resistant Staphylococcus aureus (MRSA) being most common. Polymicrobial infections consisted $28 \%$ of the cases. In one case, no bacteria were identified (Table 2).

Eight patients died after the infection within the first month and 17 patients at 1 year $(21.5 \%$ and $43 \%$ respectively). Two patients died while in hospital, and their death was attributed to infection. In comparison, in the control group the mortality rate was $4.0 \%$ at 1 month and $14.6 \%$ at 1 year. This was statistically different in both cases (chi square, $p=0.016$ and $p=0.014$ respectively) (Table 3 ). At the final follow-up, two patients were not traced and four more died after the first year, leaving 16 patients for the final evaluation. For those patients who were available the mean follow-up was 4.5 years (range 1.5 to 9.5 years). At the final follow-up VAS, Parker, Barthel and EQ-5D-5L were measured (Table 3). The control group had better functional score as measured with the Barthel index, and this difference was both statistically and clinically significant. The control group had better mobility status (Parker mobility score), less pain (VAS pain score) and better quality of life (EQ-5D-5L), but these differences did not reach significance (Table 3). Complications were more common in the infection group (41\% vs. $8.3 \%$ ) (chi-squared test, $p<0.001$ ) and included lung infections, urinary tract infections, strokes, myocardial infractions, deep vein thrombosis and acute kidney injuries.

The final functional outcome as measured with Barthel index score was affected by the previous functional state (Barthel index before hip fracture), haemoglobin at admission, age, time from admission to surgery, operative time, and the presence of dementia and infection (univariate analysis). Besides pre-fracture functional outcome, the multiple regression model showed that infection $(p=0.03)$ independently affected the final Barthel score. The model explained the $70 \%$ of the variability of the final functional outcome (Table 4). Finally, a logistic regression model was utilised to identify any risk factors that predisposed to infection. While increased operative time, time from admission to surgery, extra-capsular fracture and wound oozing at $7 \mathrm{~d}$ were found to increase the risk of infection, in the multivariate analysis only time to surgery remained a significant risk factor $(p=0.022$ ) (Table 5). The model predicted that every day of delay increases the odds ratio of infection by a factor of 1.8.

\section{Discussion}

In the present study, the infection after hip fracture in elderly patients was associated with diminished functional ability and higher mortality. This study also demonstrated that the final functional outcome of patients with hip fractures depends on the preoperative physical function and is negatively affected by the presence of infection. Delay in surgery almost doubles the risk for infection. Even if the incidence of infection is rather small, given the frequency of geriatric hip fractures and the poor outcomes of infection management, every attempt should be made to avoid this devastating complication.

The 1-year mortality in the FRI group was $44 \%$ compared to $14.6 \%$ in the control group. Duckworth et al. (2012) also found higher mortality rate in patients with deep infection after hip fractures compared to patients without infections, although not statistically significant (33\% vs. $28 \%$ ). In the same study it was reported that dementia and $S$. aureus infection were associated with increased mortality. 
Table 3. Comparison between hip fracture patients with infection and without infection. Mann-Whitney $U$ test was used for continuous variables and chi square for categorical.

\begin{tabular}{llll}
\hline & Infection group & Control group & $p$ value \\
\hline Barthel index score pre-op [(median (IQR)] & $20(1)$ & $20(1)$ & 0.872 \\
Barthel index score final [(median (IQR)] & $14(16)$ & $18(5)$ & $0.017^{*}$ \\
EQ-5D-5L index [(median (IQR)] & $0.742(0.75)$ & $0.769(0.123)$ & 0.065 \\
Parker mobility score [(median (IQR)] & $8(4)$ & $8(3)$ & 0.071 \\
VAS pain score [(median (IQR)] & $1(1)$ & $0(2)$ & 0.107 \\
Mortality at 30d [n $(\%)]$ & $8(20.5 \%)$ & $8(4.0 \%)$ & $0.016^{*}$ \\
Mortality at 1 year $[n(\%)]$ & $17(44 \%)$ & $29(14.6 \%)$ & $0.014^{*}$ \\
\hline
\end{tabular}

$* p<0.05$

Table 4. List of variables tested in the model regarding the final Barthel index score. Variables with statistically significant correlations or differences were entered in the multiple regression model.

\begin{tabular}{|c|c|c|c|c|c|}
\hline \multicolumn{6}{|l|}{ Univariate analysis } \\
\hline & \multicolumn{2}{|c|}{ Value (Barthel index score) } & \multicolumn{3}{|c|}{$p$ value } \\
\hline Sex $($ female/male $)($ mean \pm SD $)$ & \multicolumn{2}{|c|}{$16 \pm 6$ vs. $17.5 \pm 4$} & \multicolumn{3}{|c|}{0.09} \\
\hline Smoking (yes/no) (mean \pm SD) & \multicolumn{2}{|c|}{$17.4 \pm 6$ vs. $15.9 \pm 5$} & \multicolumn{3}{|c|}{0.954} \\
\hline Diabetes mellitus (yes/no) (mean \pm SD) & \multicolumn{2}{|c|}{$15.3 \pm 6$ vs. $16.7 \pm 5$} & \multicolumn{3}{|c|}{0.303} \\
\hline Dementia $($ yes/no $)($ mean $\pm S D)$ & \multicolumn{2}{|c|}{$12 \pm 7$ vs. $17 \pm 4$} & \multicolumn{3}{|c|}{0.017} \\
\hline $\begin{array}{ll}\text { Previous } & \text { accommodation } \\
\text { (home/geriatric centre) } & (\text { mean } \pm \text { SD) }\end{array}$ & \multicolumn{2}{|c|}{$16.8 \pm 5$ vs. $13.1 \pm 8$} & \multicolumn{3}{|c|}{0.056} \\
\hline $\begin{array}{l}\text { Previous mobilisation status (indepen- } \\
\text { dent/with assistance) }(\text { mean } \pm \mathrm{SD})\end{array}$ & \multicolumn{2}{|c|}{$17.2 \pm 5$ vs. $15 \pm 5.5$} & \multicolumn{3}{|c|}{0.275} \\
\hline $\begin{array}{l}\text { Use of anti-coagulants (yes/no) } \\
(\text { mean } \pm \mathrm{SD})\end{array}$ & \multicolumn{2}{|c|}{$15.7 \pm 5$ vs. $16.5 \pm 6$} & \multicolumn{3}{|c|}{0.649} \\
\hline $\begin{array}{l}\text { Fracture type (extra-/intra-capsular) } \\
(\text { mean } \pm \mathrm{SD})\end{array}$ & \multicolumn{2}{|c|}{$17.3 \pm 4$ vs. $15.2 \pm 6$} & \multicolumn{3}{|c|}{0.064} \\
\hline Infection $($ yes/no) $($ mean \pm SD) & \multicolumn{2}{|c|}{$14.1 \pm 8$ vs. $17.6 \pm 5$} & \multicolumn{3}{|c|}{0.014} \\
\hline Age & \multicolumn{2}{|c|}{ Pearson correlation $(-0.326)$} & \multicolumn{3}{|c|}{0.009} \\
\hline Operative time & \multicolumn{2}{|c|}{ Pearson correlation $(0.246)$} & \multicolumn{3}{|c|}{0.063} \\
\hline Time from admission to surgery & \multicolumn{2}{|c|}{ Pearson correlation $(-0.245)$} & \multicolumn{3}{|c|}{0.073} \\
\hline ASA score & \multicolumn{2}{|c|}{ Pearson correlation $(-0.165)$} & \multicolumn{3}{|c|}{0.212} \\
\hline Haemoglobin at admission & \multicolumn{2}{|c|}{ Pearson correlation $(0.314)$} & \multicolumn{3}{|c|}{0.023} \\
\hline Albumin at admission & \multicolumn{2}{|c|}{ Pearson correlation (0.089) } & 0.5 & & \\
\hline Pre-operative Barthel index score & Pearson & elation $(0.703)$ & $<0$ & & \\
\hline Length of stay & Pearson & ation $(-0.310)$ & 0.0 & & \\
\hline Multivariate analysis model & & & & & \\
\hline Model & Unstanc & ed coefficients & Standardised coefficients & $t$ & Significance \\
\hline & $\mathrm{B}$ & Standard error & Beta & & \\
\hline (Constant) & 0.850 & 6.590 & & 0.129 & 0.898 \\
\hline Age & -0.009 & 0.073 & -0.012 & -0.123 & 0.902 \\
\hline Infection & -1.012 & 0.445 & -0.231 & -2.276 & 0.027 \\
\hline Pre-operative Barthel score & 1.038 & 0.165 & 0.639 & 6.303 & $<0.001$ \\
\hline Type of fracture & 0.757 & 0.535 & 0.133 & 1.416 & 0.163 \\
\hline Dementia & 0.558 & 1.560 & 0.035 & 0.358 & 0.722 \\
\hline
\end{tabular}


Table 5. Comparison different pre-operative, operative and postoperative factors between infection and control group that used for the logistic regression model to explore if any of them predisposed to infection.

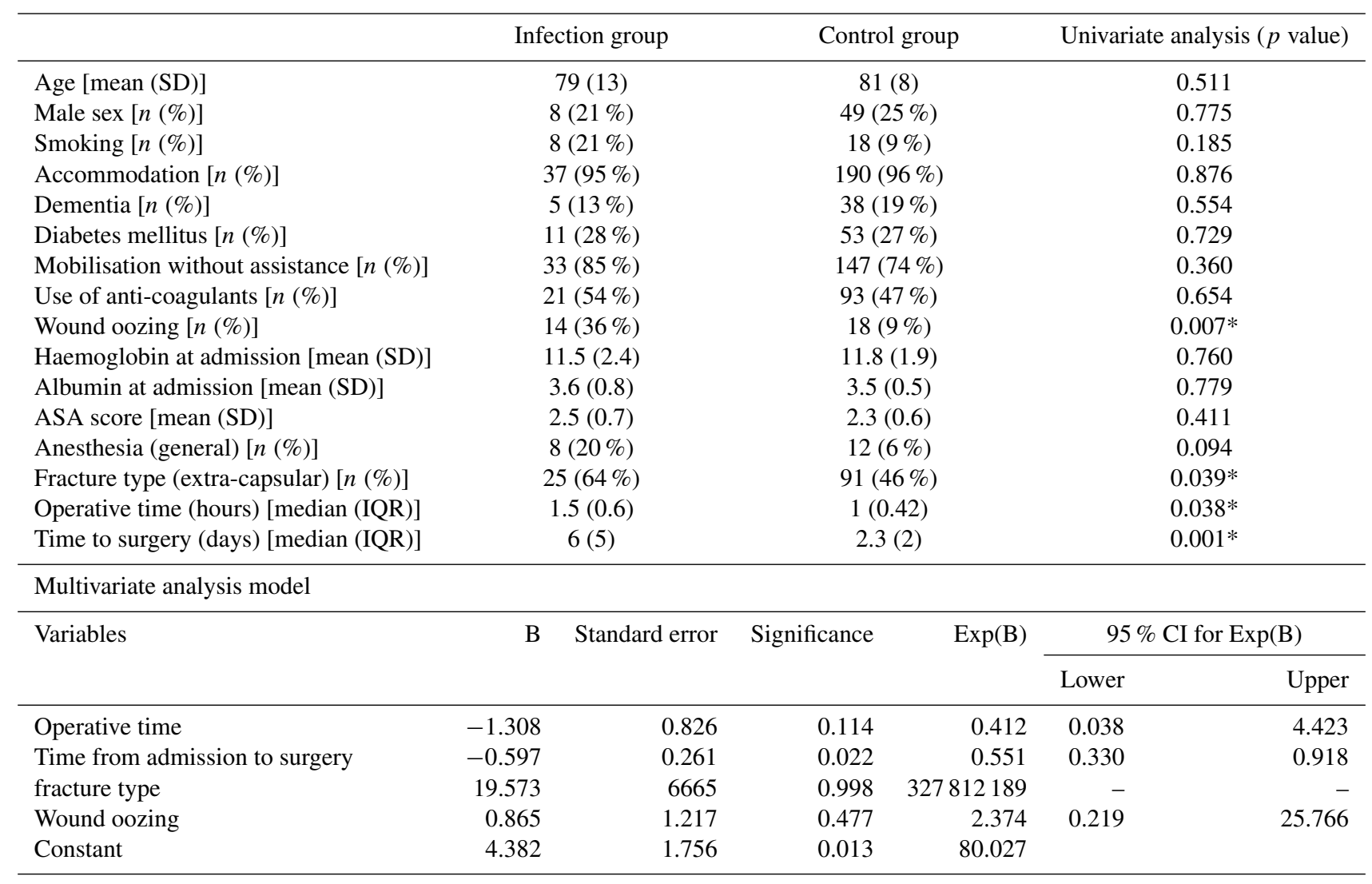

$* p<0.05$

The rate of infection in this series was $0.72 \%$, lower than the reported range of $1.2 \%$ to $3.6 \%$ (Pollard et al., 2006; Edwards et al., 2008). The most common causative microorganism is Staphylococcus aureus or Staphylococcus epidermidis, similar to the literature reports (Partanen et al., 2006; Edwards et al., 2008). Factors predisposing to fracturerelated infection after geriatric hip fractures are numerous and involve diabetes mellitus (Partanen et al., 2006), oral steroids (Edwards et al., 2008) and very long operative time (> $240 \mathrm{~min}$ ) (Edwards et al., 2008). In another recent study the rate of superficial site infection (SSI) after hip fractures reached $3.67 \%$, and diabetes, summer period, steroids therapy, increased BMI and anaemia were recognised as risk factors (Ji et al., 2019). In the current study, we found that increased operative time, delays from admission to surgery, extra-capsular fracture and wound leakage in the first $7 \mathrm{~d}$ increased the probability of infection in the univariate analysis. However, the multivariate model disclosed time from admission to surgery as the only statistically significant risk factor. This, to our knowledge, has been described in the literature for the first time. This time period does not include the time from fracture to admission, which is usually within hours after the fall causing the fracture. Given the fact that the period time from admission to surgery is a modifiable factor, every attempt should be made to shorten the time to surgery. It could be argued that the use of anticoagulants or multiple comorbidities could be confounding factors for increased likelihood of infection as they could delay the time to fracture fixation. However, the frequency of anticoagulants and the ASA score, which is a proxy for comorbidity, were comparable between the two groups. Year of infection could also be a confounding factor as the management of hip fractures differs throughout the years. Still, we observed that half of the infections occurred from 2010 to 2014 and half from 2015 to 2020, while the number of hip fractures remained stable. A longer preoperative hospitalisation may be followed by colonisation with in-hospital and usually drug-resistant bacterial flora predisposing to surgical site infections.

The functional outcome after hip fracture is compromised, and usually there is a decline in physical activity, with almost half of the patients needing to use walking aids (Thomas et al., 2010; Dyer et al., 2016). On top of this decline, the presence of infection further decreases in a clinically significant way the physical activity of patients. The median Barthel index of 14 means that these patients had severe restrictions in mobility and self-care. Few studies report on the func- 
tional outcome after infection of hip fracture management. Partanen et al. (2006) reported that patients with infection had worse walking ability and more frequently used walking aids at 4 months but did not use a validated instrument. In the present study we showed that infection predisposed to worse clinical and functional results as demonstrated by the decline in Barthel score. Moreover, besides pre-fracture functional status, which has already been recorded, our study showed that infection independently affects the physical status of patients.

The Parker mobility score showed a trend $(p=0.071)$ of being lower in patients with infections. Also, quality of life was poorer but without reaching the clinically significant difference of 0.08 or a statistical difference. A recent study disclosed a EQ-5D-5L index of 0.76 in patients with FRI of long bones, which is higher than our FRI group, probably because of the younger mean age of their cohort (60 vs. 79 years) (Walter et al., 2021). Finally, pain did not differ between groups. The reason for the discrepancy that patients complicated with infection are not experiencing worse quality of life while having worse physical function is not known.

Limitations of the study include the small number of patients. FRI after hip fracture is a rare event making data collection and analysis very difficult. In the literature, similar numbers of patients are reported, although the observation period varies from 2 to 10 years. Duckworth et al. (2012) reported on 43 patients and Edwards et al. (2008) on 41 patients in studies with the biggest numbers of patients. Also, the patients were not matched for the year of hip fracture. The control group was consecutive patients with hip fractures operated from January to June 2018 and was the best available regarding data collection. Another limitation is the fact that some cases of infection might receive treatment in another hospital. However, this seems a remote possibility as our unit is the reference centre for infections. Finally, as an observational study, confounding factors may exist. We tried to eliminate this by exploring possible confounding factors like ASA score and use of anticoagulants. Variables like steroid use or BMI was not available for all patients and were not included in the analysis. The strength of the study is that a validated instrument for assessment was used and that prospectively collected data were available.

\section{Conclusions}

Although the incidence of post-operative infection after hip fracture surgery is low, it increases mortality and imposes a significant limitation on the functional outcome. The delay to surgery increases the likelihood for infection. Therefore all physicians involved in the management of elderly with hip fractures should collaborate to develop optimisation pathways of these patients, which would allow early surgical management to mitigate the infection risk and the related consequences on the function, the mortality and the health care costs.

Ethical statement. Informed consent was obtained from all patients included in the study. The study was approved by the institutional review board (IRB no. 43194/2020). The study complied with the Declaration of Helsinki.

Data availability. Data are accessible from the electronic database of our department. Data are available from the corresponding author upon request.

Author contributions. AAK designed the study, performed the analysis and wrote the manuscript. CB and VA collected the data and helped with data analysis. CA reviewed the manuscript and helped with the data analysis. KNM designed the study and reviewed the manuscript. All authors read and approved the final manuscript.

Competing interests. The contact author has declared that neither they nor their co-authors have any competing interests.

Disclaimer. Publisher's note: Copernicus Publications remains neutral with regard to jurisdictional claims in published maps and institutional affiliations.

Review statement. This paper was edited by Bryan Springer and reviewed by two anonymous referees.

\section{References}

Bouwstra, H., Smit, E. B., Wattel, E. M., van der Wouden, J. C., Hertogh, C. M. P. M., Terluin, B., and Terwee, C. B.: Measurement Properties of the Barthel Index in Geriatric Rehabilitation, J. Am. Med. Dir. Assoc., 20, 420-425, https://doi.org/10.1016/j.jamda.2018.09.033, 2019.

Cierny 3rd, G., Mader, J. T., and Penninck, J. J.: A clinical staging system for adult osteomyelitis, Clin. Orthop. Relat. Res., 414, 724, https://doi.org/10.1097/01.blo.0000088564.81746.62, 2003.

Duckworth, A. D., Phillips, S. A., Stone, O., Moran, M., Breusch, S. J., and Biant, L. C.: Deep infection after hip fracture surgery: predictors of early mortality, Injury, 43, 1182-1186, https://doi.org/10.1016/j.injury.2012.03.029, 2012.

Dyer, S. M., Crotty, M., Fairhall, N., Magaziner, J., Beaupre, L. A., Cameron, I. D., and Sherrington, C.: A critical review of the longterm disability outcomes following hip fracture, BMC Geriatr., 16, 158, https://doi.org/10.1186/s12877-016-0332-0, 2016.

Edwards, C., Counsell, A., Boulton, C., and Moran, C. G.: Early infection after hip fracture surgery: risk factors, costs and outcome, J. Bone Joint Surg. Br., 90, 770-777, https://doi.org/10.1302/0301-620X.90B6.20194, 2008. 
Herdman, M., Gudex, C., Lloyd, A., Janssen, M., Kind, P., Parkin, D., Bonsel, G., and Badia, X.: Development and preliminary testing of the new five-level version of EQ-5D (EQ-5D-5L), Qual. Life Res., 20, 1727-1736, https://doi.org/10.1007/s11136-0119903-x, 2011.

Ji, C., Zhu, Y., Liu, S., Li, J., Zhang, F., Chen, W., and Zhang, Y.: Incidence and risk of surgical site infection after adult femoral neck fractures treated by surgery: A retrospective case-control study, Medicine (Baltimore), 98, e14882, https://doi.org/10.1097/MD.0000000000014882, 2019.

Johnell, O. and Kanis, J. A.: An estimate of the worldwide prevalence, mortality and disability associated with hip fracture, Osteoporos. Int., 15, 897-902, 2004.

Kristensen, M. T., Foss, N. B., Ekdahl, C., and Kehlet, H.: Prefracture functional level evaluated by the New Mobility Score predicts in-hospital outcome after hip fracture surgery, Acta Orthop., 81, 296-302, https://doi.org/10.3109/17453674.2010.487240, 2010.

Mahoney, F. I. and Barthel, D. W.: Functional evaluation: The Barthel index, Md. State Med. J., 14, 61-65, 1965.

Mayoral, A. P., Ibarz, E., Gracia, L., Mateo, J., and Herrera, A.: The use of Barthel index for the assessment of the functional recovery after osteoporotic hip fracture: One year follow-up, PLoS One, 4, e0212000, https://doi.org/10.1371/journal.pone.0212000, 2019

Metsemakers, W. J., Morgenstern, M., McNally, M. A., Moriarty, T. F., McFadyen, I., Scarborough, M., Athanasou, N. A., Ochsner, P. E., Kuehl, R., Raschke, M., Borens, O., Xie, Z., Velkes, S., Hungerer, S., Kates, S. L., Zalavras, C., Giannoudis, P. V., Richards, R. G., and Verhofstad, M. H. J.: Fracture-related infection: A consensus on definition from an international expert group, Injury, 49, 505-510, https://doi.org/10.1016/j.injury.2017.08.040, 2018.

Owens, W. D., Felts, J. A., and Spitznagel Jr., E. L.: ASA physical status classifications: a study of consistency of ratings, Anesthesiology, 49, 239-243, https://doi.org/10.1097/00000542197810000-00003,1978.

Parker, M. J. and Palmer, C. R.: A new mobility score for predicting mortality after hip fracture, J. Bone Joint Surg. Br., 75, 797-798, 1993.
Partanen, J., Syrjälä, H., Vähänikkilä, H., and Jalovaara, P.: Impact of deep infection after hip fracture surgery on function and mortality, J. Hosp. Infect., 62, 44-49, https://doi.org/10.1016/j.jhin.2005.04.020, 2006.

Polinder, S., van Beeck, E. F., Essink-Bot, M. L., Toet, H., Looman, C. W., Mulder, S., and Meerding, W. J.: Functional outcome at 2.5, 5, 9, and 24 months after injury in the Netherlands, J. Trauma, 62, 133-141, https://doi.org/10.1097/TA.0b013e31802b71c9, 2007.

Pollard, T. C., Newman, J. E., Barlow, N. J., Price, J. D., and Willett, K. M.: Deep wound infection after proximal femoral fracture: consequences and costs, J. Hosp. Infect., 63, 133-139, https://doi.org/10.1016/j.jhin.2006.01.015, 2006.

Sims, A. L, Parsons, N., Achten, J., Griffin, X. L., Costa, M. L., and Reed, M. R.: A randomized controlled trial comparing the Thompson hemiarthroplasty with the Exeter polished tapered stem and Unitrax modular head in the treatment of displaced intracapsular fractures of the hip: the WHiTE 3: HEMI Trial, Bone Joint J., 100-B, 352-360, https://doi.org/10.1302/0301620X.100B3.BJJ-2017-0872.R2, 2018.

Thomas, S., Halbert, J., Mackintosh, S., Cameron, I. D., Kurrle, S., Whitehead, C., Miller, M., and Crotty, M.: Walking aid use after discharge following hip fracture is rarely reviewed and often inappropriate: an observational study, J. Physiother., 56, 267-272, https://doi.org/10.1016/s1836-9553(10)70010-2, 2010.

Walter, N., Rupp, M., Hierl, K., Pfeifer, C., Kerschbaum, M., Hinterberger, T., and Alt, V.: Long-term patient-related quality of life after fracture-related infections of the long bones, Bone Joint Res., 10, 321-327, https://doi.org/10.1302/2046-3758.105.BJR2020-0532, 2021.

Walters, S. J. and Brazier, J. E.: Comparison of the minimally important difference for two health state utility measures: EQ-5D and SF-6D, Qual. Life Res., 14, 1523-1532, https://doi.org/10.1007/s11136-004-7713-0, 2005.

Xu, B. Y., Yan, S., Low, L. L., Vasanwala, F. F., and Low, S. G.: Predictors of poor functional outcomes and mortality in patients with hip fracture: a systematic review, BMC Musculoskelet. Disord., 20, 568, https://doi.org/10.1186/s12891-019-2950-0, 2019. 